\title{
Presynaptic Development at L4 to L2/3 Excitatory Synapses Follows Different Time Courses in Visual and Somatosensory Cortex
}

\author{
Claire E. J. Cheetham and Kevin Fox \\ School of Biosciences, Cardiff University, Cardiff CF10 3AX, United Kingdom
}

\begin{abstract}
Visual and somatosensory cortices exhibit profound experience-dependent plasticity during development and adulthood and are common model systems for probing the synaptic and molecular mechanisms of plasticity. However, comparisons between the two areas may be confounded by a lack of accurate information on their relative rates of development. In this study, we used whole-cell recording in acute brain slices to study synaptic development in mouse barrel and visual cortex. We found that short-term plasticity (STP) switched from strong depression at postnatal day $(\mathrm{P}) 12$ to weaker depression and facilitation in mature cortex. However, presynaptic maturation was delayed by $\sim 2$ weeks at layer $(\mathrm{L}) 4$ to L2/3 excitatory synapses in visual cortex relative to barrel cortex. This developmental delay was pathway-specific; maturation of L2/3 to L2/3 synapses occurred over similar timescales in barrel and visual cortex. The developmental increase in the paired-pulse ratio to values greater than unity was mirrored by a developmental decrease in presynaptic release probability. Therefore, L4 to L2/3 excitatory synapses had lower release probabilities and showed greater short-term facilitation in barrel cortex than in visual cortex at P28. Postsynaptic mechanisms could not account for the delayed maturation of STP in visual cortex. These findings indicate that synaptic development is delayed in the L4 to L2/3 pathway in visual cortex, and emphasize the need to take into account the changes in synaptic properties that occur during development when comparing plasticity mechanisms in different cortical areas.
\end{abstract}

\section{Introduction}

Critical period plasticity has been studied intensively in sensory cortex in an effort to understand the molecular and structural mechanisms underlying experience-dependent changes in cortical maps. The visual cortex and the barrel cortex are arguably the two most prominent model systems for understanding experiencedependent plasticity (Fox and Wong, 2005). Individual labs tend to work on one or other of these sensory systems. Nevertheless, researchers often seek to compare results across cortical areas to identify common principles of cortical function or development. Such comparisons can, however, lead to ambiguous conclusions, as differences between cortical areas could arise either because the mechanisms involved differ per se, or because the two areas are at different developmental stages at the same age. For example, the critical period for layer (L) 4 occurs at different ages in rodent barrel and visual cortex (Woolsey and Wann, 1976; Gordon and Stryker, 1996; Stern et al., 2001). It therefore seems a reasonable hypothesis that the visual and somatosensory cortices develop either at different rates or at different ages.

We sought to test this idea using the excitatory L4 to L2/3 pathway as a test case. This pathway has been implicated as being

Received May 18, 2010; revised July 1, 2010; accepted July 8, 2010.

This study was funded by the National Institute of Mental Health (Grant P50-MH077972) and the Medical Research Council.

Correspondence should be addressed to either Claire Cheetham or Kevin Fox, School of Biosciences, Cardiff University, Museum Avenue, Cardiff CF103AX, UK. E-mail: cheethamce@cf.ac.uk or foxkd@cf.ac.uk.

DOI:10.1523/JNEUROSCI.2544-10.2010

Copyright $\odot 2010$ the authors $\quad$ 0270-6474/10/3012566-06\$15.00/0 important for plasticity in the visual and somatosensory systems (Kirkwood et al., 1993; Glazewski and Fox, 1996; Allen et al., 2003; Crozier et al., 2007) and represents a major early intracortical pathway for transmission of information out of L4. We measured short-term plasticity (STP), which plays an important role in cortical information processing (Tsodyks and Markram, 1997; Abbott and Regehr, 2004), to study the postnatal development of synaptic transmission from postnatal day (P) 12 to adulthood.

We show that maturation of STP at L4 to L2/3 excitatory synapses occurs $\sim 2$ weeks later in visual cortex than in barrel cortex. Maturation involves an increase in paired-pulse ratio (PPR) and steady-state amplitude in both cortical areas, and STP converges to similar values in adult barrel and visual cortex. Developmental changes in STP are mirrored by changes in presynaptic initial release probability.

\section{Materials and Methods}

Animals. Procedures were performed in accordance with the UK Animals (Scientific Procedures) Act 1986. C57BL/6JOlaHsd mice (Harlan) at P12-P13 [P12], P26-P30 [P28], P40-P44 [P42], P56-P63 [P60], and $\mathrm{P} 113-\mathrm{P} 138$ [P125] were used. Mean ages given in square brackets are quoted for clarity. Recordings were made from 376 cells from 76 mice (male and female). One cell per slice was recorded; $\leq 3$ cells in any dataset were recorded from each mouse; each dataset comprised recordings from $\geq 3$ mice.

Brain slice preparation. Mice were killed by cervical dislocation and decapitated, and brains were rapidly removed and placed in ice-cold dissection buffer (in mm: 108 choline-Cl, $3 \mathrm{KCl}, 26 \mathrm{NaHCO}_{3}, 1.25$ $\mathrm{NaH}_{2} \mathrm{PO}_{4}, 25$ D-glucose, $3 \mathrm{Na}$-pyruvate, $1 \mathrm{CaCl}_{2}, 6 \mathrm{MgSO}_{4}, 285 \mathrm{mOsm}$ ) 
bubbled with $95 \% \mathrm{O}_{2} / 5 \% \mathrm{CO}_{2}$. Coronal slices (400 $\mu \mathrm{m}$ thick) were cut using a VT1000S microtome (Leica Microsystems). Brain slices were incubated in artificial CSF (ACSF; in mM: $119 \mathrm{NaCl}, 3.5 \mathrm{KCl}, 1 \mathrm{NaH}_{2} \mathrm{PO}_{4}$, 10 D-glucose, $2 \mathrm{CaCl}_{2}, 1 \mathrm{MgSO}_{4}, 300 \mathrm{mOsm}$ ) bubbled with $95 \% \mathrm{O}_{2} / 5 \%$ $\mathrm{CO}_{2}$, at $32^{\circ} \mathrm{C}$ for $45 \mathrm{~min}$, then at room temperature until recording. Recordings were made in barrel and visual cortex slices from the same mice on the same days.

Whole-cell recording. Slices containing either primary visual cortex or a continuous band of barrels were identified under bright-field transillumination using an Olympus BX50WI microscope. Recordings in visual cortex were targeted to the binocular zone using anatomical landmarks as a guide (Franklin and Paxinos, 2008). L2/3 pyramidal neurons were identified under infrared differential interference contrast. Whole-cell voltage recordings were made at $35-37^{\circ} \mathrm{C}$. Recording pipettes $(5-8 \mathrm{M} \Omega$ ) contained the following (in mM): $130 \mathrm{KMeSO}_{4}, 8 \mathrm{NaCl}, 2 \mathrm{KH}_{2} \mathrm{PO}_{4}, 2$ D-glucose, 10 HEPES, $4 \mathrm{Mg}$-ATP, 7 phosphocreatine, 0.3 GTP, 0.5 ADP, $\mathrm{pH}$ 7.30, 285 mOsm. Recorded neurons had resting membrane potentials (Vm; not corrected for liquid junction potential) $\leq-70 \mathrm{mV}$, except for P12 neurons ( $\mathrm{Vm} \leq-60 \mathrm{mV})$. No holding current was used. The identity of pyramidal neurons was confirmed by regular spiking behavior in response to depolarizing current injection. Recordings were discarded if $\mathrm{Vm}$ depolarized by $>5 \mathrm{mV}$ or access resistance (15-35 M $\Omega$ ) increased by $>20 \%$. Voltage-clamp recordings were not performed, as nonlinearities that arise due to imperfect space clamp (Williams and Mitchell, 2008) could alter STP.

A tungsten monopolar extracellular stimulating electrode (0.5 M $\Omega$ ) was positioned either vertically above the recorded neuron in L4 or in $\mathrm{L} 2 / 3$ of the adjacent barrel column or the equivalent distance separation within visual cortex. The distance between stimulating and recording electrodes in L2/3 differed between age groups but not between cortical areas $(234 \pm 12 \mu \mathrm{m}$ at $\mathrm{P} 12 ; 291 \pm 18 \mu \mathrm{m}$ at $\mathrm{P} 28 ; p=0.004$ for effect of age, $p>0.05$ for effect of cortical area, two-way ANOVA). Paired recordings were not feasible due to the low connectivity of long-range intracortical pathways. Extracellular stimuli consisted of $1 \mathrm{~ms}$ current pulses with stimulation intensity $(0.8-4.0 \mathrm{~V})$ set to produce a 3-6 $\mathrm{mV}$ monosynaptic EPSP in the postsynaptic neuron. Peaks of monosynaptic EPSPs were within $5 \mathrm{~ms}$ of stimulus onset. Access resistance and pipette capacitance were compensated and data were amplified (Multiclamp 700B; Molecular Devices). Postsynaptic responses were low-pass filtered at $6 \mathrm{kHz}$, digitized at $10-20 \mathrm{kHz}$ and recorded using Signal v1.85/v4.01 (Cambridge Electronic Devices). The relationship between stimulus voltage and evoked EPSP amplitude was very similar at different ages and between cortical areas (supplemental Table 1, available at www.jneurosci. org as supplemental material). Input-output curves were not performed because the high stimulus intensities required to generate them resulted in significant polysynaptic activation for $\mathrm{P} 12$ neurons even in highdivalent ACSF ( $4 \mathrm{~mm} \mathrm{CaCl}_{2}$ and $4 \mathrm{~mm} \mathrm{MgSO}_{4}$ ).

STP. Neurons were stimulated with $\geq 20$ trains of eight pulses (Master8; AMPI) delivered at $20 \mathrm{~Hz}$, with $15 \mathrm{~s}$ between trials. NMDA receptor (NMDAR)-mediated transmission was blocked by bath application of $50 \mu \mathrm{M}$ D-AP5, and AMPA receptor (AMPAR) desensitization by bath application of $50 \mu \mathrm{M}$ cyclothiazide and $80 \mu \mathrm{M}$ PEPA (2-[2,6-difluoro-4[[2-[(phenylsulfonyl)amino]ethyl] thio]phenoxy]acetamide) (all from Tocris Bioscience). Inhibition onto L2/3 pyramidal neurons was blocked by inclusion of $1 \mathrm{~mm}$ picrotoxin in the pipette internal solution, allowing 15 min equilibration time before data acquisition.

Monosynaptic EPSP amplitudes were measured using Signal, as the voltage difference between baseline (before response onset) and peak membrane potentials. Initial analysis comparing STP measurements based on EPSP amplitude and EPSP slope showed no differences (supplemental Fig. 1, available at www.jneurosci.org as supplemental material). Therefore STP was assessed by calculating the PPR (EPSP2/ EPSP1 amplitude ratio) and the normalized steady-state amplitude (mean EPSP7-EPSP8 amplitude normalized to EPSP1 amplitude) for each neuron.

Rate of use-dependent blockade of NMDARs by MK-801. NMDARmediated responses were isolated in $\mathrm{Mg}^{2+}$-free ACSF containing $20 \mu \mathrm{M}$ CNQX (6-cyano-7-nitroquinoxaline-2,3-dione). Single presynaptic stimuli were delivered at $0.1 \mathrm{~Hz}$. Stable baseline responses $(\sim 5 \mathrm{mV})$ were
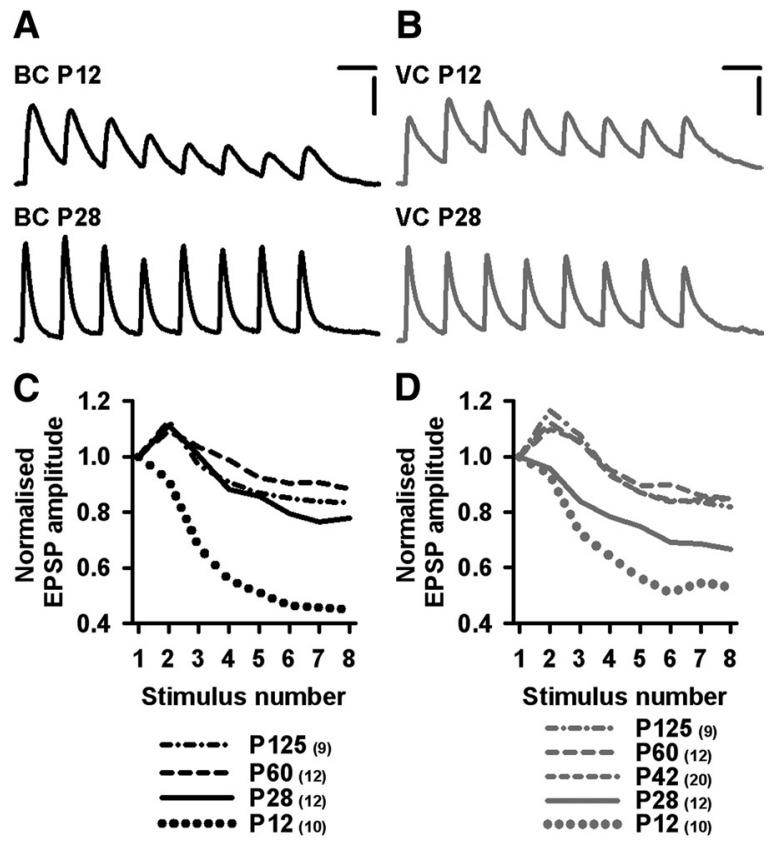

E
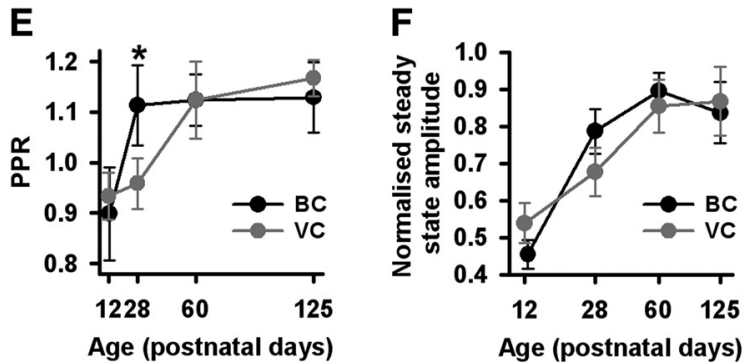

Figure 1. Maturation of STP at $L 4-L 2 / 3$ excitatory synapses occurs later in visual cortex than in barrel cortex. $\boldsymbol{A}$, Responses to $20 \mathrm{~Hz}$ stimulus trains in barrel cortex (BC) neurons (average of 20 trials). $\boldsymbol{B}$, As in $\boldsymbol{A}$, but for neurons in visual cortex (VC). Scale bars, $2 \mathrm{mV} / 50$ ms. $C$, Maturation of STP in barrel cortex. $p<0.001$ for $P 12$ versus $P 28, p>0.21$ for other pairwise comparisons of successive age groups; tw0-way ANOVA with Bonferroni correction of responses to stimuli $2-8$ ( $p<0.001$ for age and stimulus number). $D$, Maturation of STP in visual cortex. $p=0.013$ for $\mathrm{P} 12$ versus $\mathrm{P} 28, p<0.001$ for $\mathrm{P} 28$ versus $\mathrm{P} 42, p=$ 1.00 for other pairwise comparisons of successive age groups; two-way ANOVA as in $C$ $(p<0.001$ for age and stimulus number). $\boldsymbol{E}$, Change in PPR with age in barrel cortex and visual cortex. ${ }^{*} p=0.046$, P2 28 barrel cortex versus visual cortex, $t$ test. $\boldsymbol{F}$, Change in normalized steady-state amplitude with age in barrel cortex and visual cortex. $p=0.24$, P28 barrel cortex versus visual cortex, $t$ test.

recorded before MK-801 application. Stimulation was halted for $10 \mathrm{~min}$ during $10 \mu \mathrm{M}$ MK-801 wash-on, then resumed at $0.1 \mathrm{~Hz}$. Response amplitudes to successive stimuli were normalized to the first response after MK-801 application. Response curves were fitted with double exponential functions.

Measurement of evoked responses in strontium. Recordings were made in $\mathrm{Ca}^{2+}$-free ACSF containing $4 \mathrm{~mm} \mathrm{MgSO}_{4}$ and $4 \mathrm{~mm} \mathrm{SrCl}_{2}$. $\mathrm{L} 4$ axons were stimulated at $0.01 \mathrm{~Hz}$, with stimulus intensity set to evoke initial synchronous EPSPs of $\sim 2 \mathrm{mV}$. Evoked EPSPs in strontium (Sr-EPSPs) that occurred 150-350 ms after stimulus onset were identified and measured manually using Signal; this time window minimized contamination either by the decay phase of the initial synchronous EPSP or by spontaneous EPSPs. At least $60 \mathrm{Sr}$-EPSPs per cell were measured.

Statistics. Data are shown as mean \pm SEM, except for STP data in Figures $1, C$ and $D$, and $2 B$, which are shown as mean values for clarity. Data that passed tests for normality and equal variance were analyzed with parametric statistical tests; nonparametric tests were used for other analyses. All tests were two tailed with $\alpha=0.05$. 


\section{Results}

Maturation of STP at L4 to L2/3

excitatory synapses is delayed in visual cortex relative to barrel cortex

To investigate developmental changes in STP at L4 to L2/3 excitatory synapses in barrel and visual cortex, we made wholecell voltage recordings of $\mathrm{L} 2 / 3$ pyramidal neurons at $35-37^{\circ} \mathrm{C}$ in acute brain slices. Axons in L4 were stimulated with eightpulse trains at $20 \mathrm{~Hz}$ (Fig. $1 A, B$ ). At P12, EPSPs depressed throughout the stimulus train in both cortical areas (Fig. 1C,D). In barrel cortex, the pattern of STP changed significantly between P12 and P28 (Fig. $1 C)$, exhibiting developmental increases in both PPR ( $p=0.045$, Mann-Whitney rank sum test) and steady-state amplitude $(p<0.001, t$ test) (Fig. $1 E)$. Mean response profiles were very similar at P28, P60, and P125 (Fig. 1C), indicating that STP at L4 to L2/3 excitatory synapses is largely mature by P28 in barrel cortex.

In contrast, in visual cortex there was no significant change in $\operatorname{PPR}(p=0.73, t$ test) or steady-state amplitude $(p=0.16$, $t$ test) between P12 and P28 (Fig. 1D-F). Therefore, PPR was significantly larger in barrel cortex than in visual cortex at P28 ( $p=0.046, t$ test), although steady-state amplitudes in the two areas were not significantly different $(p=$ $0.24, t$ test). However, PPR increased dramatically between P28 and P42 in visual cortex ( $p=0.024, t$ test $)$ and steady-state amplitude also increased to a value similar to that seen in mature barrel cortex. The mean response profile was very similar in the $\mathrm{P} 42, \mathrm{P} 60$, and $\mathrm{P} 125$ groups, indicating that STP is mature in visual cortex by P42; this mature pattern of STP was also very similar to that seen in mature barrel cortex (Fig. $1 C-F$ ). In summary, maturation of STP at L4 to L2/3 excitatory synapses occurs $\sim 2$ weeks later in visual cortex than in barrel cortex.

Time course of maturation of STP at horizontal L2/3 to L2/3 excitatory synapses is similar in barrel and visual cortex We next investigated whether delayed maturation of STP is a general property of excitatory inputs to $\mathrm{L} 2 / 3$ pyramidal neurons by studying their long-range horizontal inputs (Fig. $2 \mathrm{~A}$ ). There was a marked change in the mean response profile in both cortical areas between P12 and P28 (Fig. 2 B). Both PPR and steady-state amplitude increased significantly between P12 and P28, but there was no difference in either parameter between barrel and visual cortex at either age (Fig. $2 C, D$ ). Therefore, delayed maturation of STP in visual cortex is specific to the $\mathrm{L} 4$ to $\mathrm{L} 2 / 3$ pathway.

Developmental changes in initial presynaptic release probability mirror developmental changes in PPR

We next investigated presynaptic release probability at L4 to L2/3 excitatory synapses in barrel and visual cortex during the first postnatal month, using the rate of use-dependent blockade of NMDARs by the irreversible open-channel blocker MK-801 (Hessler et al., 1993; Rosenmund et al., 1993) (Fig. 3A). This measure is directly related to initial release probability, as channel
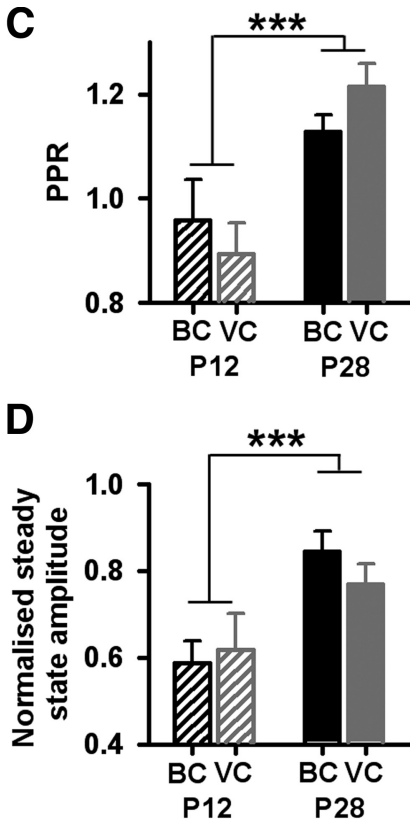

Figure 2. Time course of maturation of STP at long-range L2/3-L2/3 synapses is similar in barrel and visual cortex. $\boldsymbol{A}$, Responses 作 — VC P28 (10) .
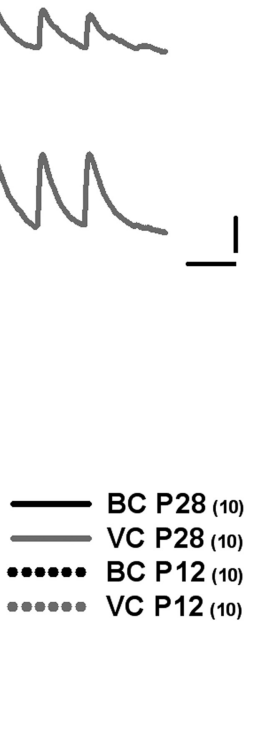

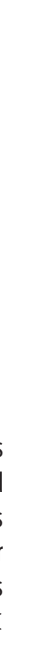

blockade is dependent on channel opening, which is in turn dependent on neurotransmitter release and binding. Usedependent blockade occurred at similar rates in barrel and visual cortex at P12 (Fig. 3B), but was significantly faster in visual cortex than in barrel cortex at P28 (Fig. 3C). The rate of use-dependent blockade decreased significantly between P12 and P28 in barrel cortex ( $p<0.001$ for effect of age, two-way ANOVA), but not in visual cortex ( $p=0.20$ for effect of age, two-way ANOVA) (Fig. $3 D$ ). Developmental changes in NMDAR subunit composition could not explain the different rates of use-dependent blockade in barrel and visual cortex at P28 (supplemental Fig. 2 and supplemental Discussion, available at www.jneurosci.org as supplemental material). Our data therefore show a developmental decrease in presynaptic release probability between P12 and P28 in barrel cortex, which is absent in visual cortex.

Postsynaptic mechanisms and inhibition do not contribute to the difference in STP in barrel and visual cortex at P28

We found no evidence that mechanisms other than presynaptic function contributed to the difference in STP in barrel and visual cortex at P28. Blocking AMPAR desensitization (supplemental Fig. 3, available at www.jneurosci.org as supplemental material) or NMDAR-mediated transmission or inhibition (supplemental Fig. 4, available at www.jneurosci.org as supplemental material) had no effect on STP. The postsynaptic response amplitude of L4 to L2/3 synapses, determined by recording EPSPs in the presence of extracellular strontium, which causes asynchronous presynaptic release, decreased significantly between P12 and P28 in both barrel and visual cortex (supplemental Fig. 5, available at www. jneurosci.org as supplemental material). Sr-EPSP amplitude was similar in barrel and visual cortex at P12, but was slightly larger in visual cortex than in barrel cortex at P28. There were also no differences in the resting membrane potential (barrel, $-75 \pm 1$ 
A

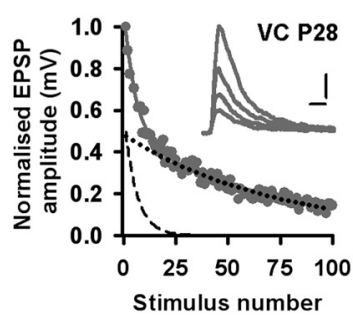

C

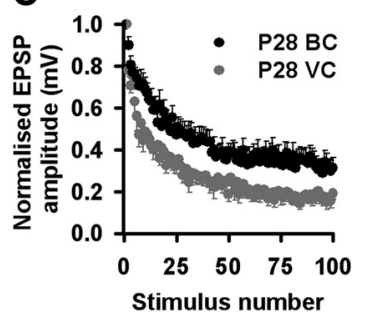

B

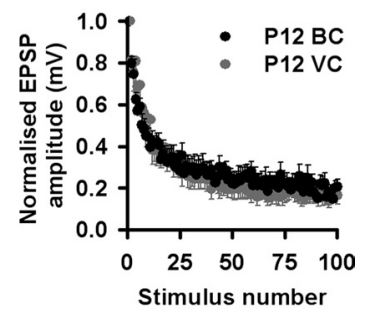

D

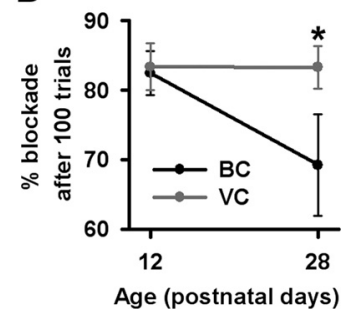

Figure 3. Lower presynaptic release probability in barrel cortex than in visual cortex at P28. A, Double-exponential fit to use-dependent blockade of NMDAR-mediated synaptic transmission by MK-801 neuron. Gray line shows double-exponential fit to data; black dashed and dotted lines indicate fast- and slow-single exponential components, respectively. Inset, Responses to first, 10th, 20th, and 50th stimuli. Scale bars, $1 \mathrm{mV} / 20 \mathrm{~ms}$. B, Rate of use-dependent blockade of NMDAR-mediated synaptic transmission by MK-801 at P12. $p=0.30$ barrel cortex $(B C)$ versus visual cortex (VC), $p<0.001$ for stimulus number, two-way ANOVA; $n=10$ per group. $\boldsymbol{C}$, As in $\boldsymbol{B}$ but at $P 28 . p<0.001$ barrel cortex versus visual cortex, $p<0.001$ for stimulus number, two-way ANOVA; $n=15$ per group. $\boldsymbol{D}$, Effect of age on percentage blockade after 100 trials. ${ }^{*} p=0.030, t$ test comparing responses $96-100$ at P28; $p=0.86, t$ test comparing responses $96-100$ at $\mathrm{P} 12$.

$\mathrm{mV}$; visual, $-75 \pm 1 \mathrm{mV} ; p=0.62, t$ test) or input resistance (barrel, $42 \pm 3 \mathrm{M} \Omega$; visual, $46 \pm 3 \mathrm{M} \Omega ; p=0.39, t$ test) of L2/3 pyramidal neurons in barrel and visual cortex at P28 $(n=27$ per group).

\section{Discussion}

Timing of presynaptic maturation in barrel and visual cortex The main finding of this study is a pathway-specific delay in presynaptic maturation at excitatory L4 to L2/3 synapses in visual cortex relative to barrel cortex. Developmental changes in STP have not previously been studied at L4 or long-range L2/3 inputs to $\mathrm{L} 2 / 3$ pyramidal neurons, or indeed in any other intracortical pathway in visual cortex. However, maturation of STP at several other excitatory intracortical connections in barrel cortex, and at local excitatory connections in L2/3 of auditory cortex, also occurs during the third and fourth postnatal weeks (Reyes and Sakmann, 1999; Frick et al., 2007; Oswald and Reyes, 2008; Feldmeyer and Radnikow, 2009). Delayed presynaptic maturation therefore appears to be specific to L4 to L2/3 synapses in visual cortex. The L4 to L2/3 pathway is the first step in intracortical information flow, is strictly feedforward, and provides the most powerful source of excitatory input to L2/3 pyramidal neurons (Chisum and Fitzpatrick, 2004), all of which make it an ideal control point for the spread of cortical excitation. Moreover, this pathway is known to play an important role in experiencedependent plasticity in both barrel and visual cortex (Kirkwood et al., 1993; Glazewski and Fox, 1996; Allen et al., 2003; Crozier et al., 2007).

The pattern of STP at L4 to L2/3 synapses in barrel cortex at P12 that we found was very similar to that observed with paired recordings of L4 to L2/3 connections at P17-P23 (Feldmeyer et al., 2002), which may suggest that in barrel cortex, developmental

changes in STP occur primarily during the third postnatal week in this pathway. At local L2/3 connections in barrel cortex, one paired recording study performed at near-physiological temperatures (Reyes and Sakmann, 1999) found facilitation similar to our results at long-range connections in L2/3, whereas another performed at room temperature (Atzori et al., 2001) described strong depression. The most likely explanation for this discrepancy is the difference in recording temperature between studies (supplemental Fig. 6, available at www.jneurosci.org as supplemental material) (Klyachko and Stevens, 2006).

\section{Mechanisms underlying maturation of STP}

Previous studies have shown an inverse relationship between initial release probability and PPR (Dobrunz and Stevens, 1997; Tsodyks and Markram, 1997; Murthy et al., 2001; Zucker and Regehr, 2002). Experience-dependent increases in short-term depression have also been linked to increased synaptic strength, defined as the proportion of available synaptic resources used to generate an EPSP (Finnerty et al., 1999). Our data strongly link developmental changes in PPR to initial release probability. However, developmental changes in steady-state amplitude were less closely related to release probability.

STP is not a unitary process; both facilitation and depression contribute over a timescale of hundreds of milliseconds (Dobrunz and Stevens, 1997; Zucker and Regehr, 2002) and often coexist even at individual synapses (Markram et al., 1998a; Abbott and Regehr, 2004). Facilitation, which depends on accumulation of residual calcium in the presynaptic terminal and cooperative calcium binding to synaptotagmins, and therefore is linked to release probability (Zucker and Regehr, 2002), is likely to have its strongest effect on PPR (Fig. 1C,D). Therefore, maturation of presynaptic calcium buffering and vesicle-release machinery (Atwood and Karunanithi, 2002) probably underlies developmental changes in PPR.

In contrast, steady-state amplitude is probably determined primarily by depression. Vesicle depletion-mediated depression would be expected to occur faster at high-release-probability synapses (Dobrunz and Stevens, 1997). However, the size of vesicle pools, vesicle-recycling mechanisms, and recovery from releasesite refractoriness, all of which contribute to depression and therefore to steady-state amplitude, and all of which are developmentally regulated, are independent of release probability (Thomson, 2000; Zucker and Regehr, 2002; Feldmeyer and Radnikow, 2009). The involvement of these distinct mechanisms can therefore explain the weaker relationship between initial release probability and steady-state amplitude.

\section{Comparison of plasticity studies in barrel and visual cortex in} the context of developmental timing

We have identified a clear delay in the maturation of presynaptic function in visual cortex, which may explain apparent discrepancies in the literature when comparing visual and somatosensory cortex. One such inconsistency concerns endocannabinoid (eCB)-dependent cortical plasticity. Experience-dependent plasticity in L2/3 of barrel cortex in vivo is blocked by treatment with the eCB antagonist AM251 at P13-P16, but not P26-P30 (Li et al., 2009). In contrast, AM251 treatment at up to P31 prevented ocular dominance shifts in L2/3 of visual cortex (Liu et al., 2008). A similar pattern is seen for eCB-dependent long-term depression (eCB-LTD), which is expressed presynaptically: eCB-LTD at $\mathrm{L} 4$ to $\mathrm{L} 2 / 3$ synapses is only present in barrel cortex up to $\sim \mathrm{P} 20$ (Bender et al., 2006; Hardingham et al., 2008), whereas it persists at least until P28 in visual cortex (Crozier et al., 2007). Differences 
in LTD-induction protocols between studies could explain the eCB-LTD data. However, our findings suggest that the reason for these discrepancies is that synapses in barrel and visual cortex are at different developmental stages at the same age.

Apparent differences in experience-dependent changes in NMDAR subunit composition between visual and barrel cortex could also be explained by different timing of synaptic development in the two areas. In barrel cortex, the composition of NMDARs changes during the first two postnatal weeks, from largely NR2B-dominated receptors to receptors that include the NR2A subunit (Flint et al., 1997; Lu et al., 2001). In contrast, in visual cortex, the change in NR2A/NR2B subunit ratio continues until $\sim 4$ weeks of age (Quinlan et al., 1999). This may explain why visual deprivation alters NMDAR kinetics at P26-P31 in visual cortex (Philpot et al., 2001), whereas whisker deprivation at similar ages has no effect on NMDAR kinetics in barrel cortex (Hardingham et al., 2008). In conclusion, our findings emphasize the need to take developmental changes in synaptic properties into account to distinguish between modulation of experience-dependent development and activity-dependent changes to synapses.

\section{Functional significance of delayed presynaptic maturation}

The difference in STP between synapses in barrel and visual cortex at P28 will profoundly affect processing of sensory inputs in the two areas (Abbott and Regehr, 2004). At this age, strong depression in visual cortex will favor the detection of stimulus onsets, whereas facilitation in barrel cortex enables synapses to transmit a rate code (Markram et al., 1998b). However, the delay in presynaptic development is unlikely to be due to fundamental differences in information processing between sensory cortices, because STP converges to a very similar pattern at adult L4 to L2/3 synapses in barrel and visual cortex. The timing of presynaptic maturation does, however, coincide with the end of developmental critical periods in barrel, visual, and auditory cortices (Fig. 1) (Oswald and Reyes, 2008). In visual cortex, critical period timing is controlled by the maturation of inhibition (Hensch, 2005) and recent evidence suggests that inhibitory changes could play a similarly important role in barrel cortex (Nowicka et al., 2009). We suggest that during early postnatal development, when inhibition is weak, strong depression of excitatory synapses is necessary as a control mechanism to prevent positive feedback excitation. Maturation of inhibition alleviates this requirement for strong depression, freeing excitatory synapses to adopt a wider range of coding strategies (Tsodyks and Markram, 1997; Abbott and Regehr, 2004).

\section{References}

Abbott LF, Regehr WG (2004) Synaptic computation. Nature 431:796-803. Allen CB, Celikel T, Feldman DE (2003) Long-term depression induced by sensory deprivation during cortical map plasticity in vivo. Nat Neurosci 6:291-299.

Atwood HL, Karunanithi S (2002) Diversification of synaptic strength: presynaptic elements. Nat Rev Neurosci 3:497-516.

Atzori M, Lei S, Evans DI, Kanold PO, Phillips-Tansey E, McIntyre O, McBain CJ (2001) Differential synaptic processing separates stationary from transient inputs to the auditory cortex. Nat Neurosci 4:1230-1237.

Bender VA, Bender KJ, Brasier DJ, Feldman DE (2006) Two coincidence detectors for spike timing-dependent plasticity in somatosensory cortex. J Neurosci 26:4166-4177.

Chisum HJ, Fitzpatrick D (2004) The contribution of vertical and horizontal connections to the receptive field center and surround in V1. Neural Netw 17:681-693.

Crozier RA, Wang Y, Liu CH, Bear MF (2007) Deprivation-induced synaptic depression by distinct mechanisms in different layers of mouse visual cortex. Proc Natl Acad Sci U S A 104:1383-1388.
Dobrunz LE, Stevens CF (1997) Heterogeneity of release probability, facilitation, and depletion at central synapses. Neuron 18:995-1008.

Feldmeyer D, Radnikow G (2009) Developmental alterations in the functional properties of excitatory neocortical synapses. J Physiol 587:18891896.

Feldmeyer D, Lübke J, Silver RA, Sakmann B (2002) Synaptic connections between layer 4 spiny neurone-layer $2 / 3$ pyramidal cell pairs in juvenile rat barrel cortex: physiology and anatomy of interlaminar signalling within a cortical column. J Physiol 538:803-822.

Finnerty GT, Roberts LS, Connors BW (1999) Sensory experience modifies the short-term dynamics of neocortical synapses. Nature 400: 367-371.

Flint AC, Maisch US, Weishaupt JH, Kriegstein AR, Monyer H (1997) NR2A subunit expression shortens NMDA receptor synaptic currents in developing neocortex. J Neurosci 17:2469-2476.

Fox K, Wong RO (2005) A comparison of experience-dependent plasticity in the visual and somatosensory systems. Neuron 48:465-477.

Franklin KBJ, Paxinos, G. (2008) The mouse brain in stereotaxic coordinates, 3rd Edition. San Diego: Academic.

Frick A, Feldmeyer D, Sakmann B (2007) Postnatal development of synaptic transmission in local networks of L5A pyramidal neurons in rat somatosensory cortex. J Physiol 585:103-116.

Glazewski S, Fox K (1996) Time course of experience-dependent synaptic potentiation and depression in barrel cortex of adolescent rats. J Neurophysiol 75:1714-1729.

Gordon JA, Stryker MP (1996) Experience-dependent plasticity of binocular responses in the primary visual cortex of the mouse. J Neurosci 16:3274-3286.

Hardingham N, Wright N, Dachtler J, Fox K (2008) Sensory deprivation unmasks a PKA-dependent synaptic plasticity mechanism that operates in parallel with CaMKII. Neuron 60:861-874.

Hensch TK (2005) Critical period plasticity in local cortical circuits. Nat Rev Neurosci 6:877-888.

Hessler NA, Shirke AM, Malinow R (1993) The probability of transmitter release at a mammalian central synapse. Nature 366:569-572.

Kirkwood A, Dudek SM, Gold JT, Aizenman CD, Bear MF (1993) Common forms of synaptic plasticity in the hippocampus and neocortex in vitro. Science 260:1518-1521.

Klyachko VA, Stevens CF (2006) Temperature-dependent shift of balance among the components of short-term plasticity in hippocampal synapses. J Neurosci 26:6945-6957.

Li L, Bender KJ, Drew PJ, Jadhav SP, Sylwestrak E, Feldman DE (2009) Endocannabinoid signaling is required for development and critical period plasticity of the whisker map in somatosensory cortex. Neuron 64:537-549.

Liu CH, Heynen AJ, Shuler MG, Bear MF (2008) Cannabinoid receptor blockade reveals parallel plasticity mechanisms in different layers of mouse visual cortex. Neuron 58:340-345.

Lu HC, Gonzalez E, Crair MC (2001) Barrel cortex critical period plasticity is independent of changes in NMDA receptor subunit composition. Neuron 32:619-634.

Markram H, Pikus D, Gupta A, Tsodyks M (1998a) Potential for multiple mechanisms, phenomena and algorithms for synaptic plasticity at single synapses. Neuropharmacology 37:489-500.

Markram H, Gupta A, Uziel A, Wang Y, Tsodyks M (1998b) Information processing with frequency-dependent synaptic connections. Neurobiol Learn Mem 70:101-112.

Murthy VN, Schikorski T, Stevens CF, Zhu Y (2001) Inactivity produces increases in neurotransmitter release and synapse size. Neuron 32:673-682.

Nowicka D, Soulsby S, Skangiel-Kramska J, Glazewski S (2009) Parvalbumin-containing neurons, perineuronal nets and experiencedependent plasticity in murine barrel cortex. Eur J Neurosci 30:20532063.

Oswald AM, Reyes AD (2008) Maturation of intrinsic and synaptic properties of layer $2 / 3$ pyramidal neurons in mouse auditory cortex. J Neurophysiol 99:2998-3008.

Philpot BD, Sekhar AK, Shouval HZ, Bear MF (2001) Visual experience and deprivation bidirectionally modify the composition and function of NMDA receptors in visual cortex. Neuron 29:157-169. 
Quinlan EM, Olstein DH, Bear MF (1999) Bidirectional, experiencedependent regulation of $N$-methyl-D-aspartate receptor subunit composition in the rat visual cortex during postnatal development. Proc Natl Acad Sci U S A 96:12876-12880.

Reyes A, Sakmann B (1999) Developmental switch in the short-term modification of unitary EPSPs evoked in layer 2/3 and layer 5 pyramidal neurons of rat neocortex. J Neurosci 19:3827-3835.

Rosenmund C, Clements JD, Westbrook GL (1993) Nonuniform probability of glutamate release at a hippocampal synapse. Science 262:754-757.

Stern EA, Maravall M, Svoboda K (2001) Rapid development and plasticity of layer 2/3 maps in rat barrel cortex in vivo. Neuron 31:305-315.
Thomson AM (2000) Molecular frequency filters at central synapses. Prog Neurobiol 62:159-196.

Tsodyks MV, Markram H (1997) The neural code between neocortical pyramidal neurons depends on neurotransmitter release probability. Proc Natl Acad Sci U S A 94:719-723.

Williams SR, Mitchell SJ (2008) Direct measurement of somatic voltage clamp errors in central neurons. Nat Neurosci 11:790-798.

Woolsey TA, Wann JR (1976) Areal changes in mouse cortical barrels following vibrissal damage at different postnatal ages. J Comp Neurol 170:53-66.

Zucker RS, Regehr WG (2002) Short-term synaptic plasticity. Annu Rev Physiol 64:355-405. 